\title{
Chapter 5. Treatment with antihypertensive drugs
}

\author{
Hypertension Research (2014) 37, 291-300; doi:10.1038/hr.2014.8
}

\section{POINT 5A}

1. The preventive effects of antihypertensive drugs on cardiovascular disease are determined by the degree to which blood pressure decreases rather than its class (Recommendation grade: A, Evidence level: I).

2. Appropriate antihypertensive drugs should be selected considering compelling indications, contraindications and conditions that require the careful use of drugs and the presence or absence of complications (Recommendation grade: $\mathbf{B}$, Evidence level: II).

3. In hypertensive patients without compelling indications, the antihypertensive drug to be first administered should be selected from $\mathrm{Ca}$ channel blockers, angiotensin-receptor blockers (ARBs), angiotensin-converting enzyme (ACE) inhibitors and diuretics (Recommendation grade: A, Evidence level: I).

4. Antihypertensive drugs are administered once a day, in principle, but as it is more important to control the blood pressure over $24 \mathrm{~h}$ splitting the dose into twice a day is desirable in some situations (Recommendation grade: $\mathbf{C} 1$, Evidence level: III).

5. A gradual reduction in blood pressure is desirable in hypertensive patients in general, but the target control level should be achieved within several weeks in high-risk patients, such as those with grade III hypertension and multiple risk factors (Recommendation grade: C1, Evidence level: III).

6. The use of two or three drugs in combination is often necessary to achieve the target of blood pressure control (Recommendation grade: A, Evidence level: I).

7. Combination therapy with different classes of antihypertensive drugs exhibits potent hypotensive effects, and is useful for achieving the target of blood pressure control (Recommendation grade: A, Evidence level: I).

8. Among the combinations of two drugs, those of a reninangiotensin (RA) system inhibitor (ACE inhibitor or ARB) + $\mathrm{Ca}$ channel blocker, RA system inhibitor + diuretic and $\mathrm{Ca}$ channel blocker + diuretic are recommended. (Recommendation grade: B, Evidence level: II)

9. Simplification of the prescription using fixed-combination drugs is useful for improving adherence and controlling blood pressure (Recommendation grade: A, Evidence level: I).

\section{BASIC PRINCIPLES FOR THE SELECTION OF ANTIHYPERTENSIVE DRUGS}

As blood pressure increases, it is more difficult to control it at the target level through lifestyle modifications alone, and treatment with antihypertensive drugs becomes necessary. The occurrence of cardiovascular disease can be prevented by reducing the blood pressure with antihypertensive drugs. Meta-analyses of large-scale clinical studies have shown that this effect is proportionate to the degree of decrease in blood pressure rather than the class of antihypertensive drug. ${ }^{226,402}$ The antihypertensive drug with the greatest hypotensive effect and suited for various accompanying conditions should be selected for each hypertensive patient.

\section{1) First-choice drugs}

Five classes of antihypertensive drugs including Ca channel blockers, ARBs, ACE inhibitors, diuretics and $\beta$-blockers (including $\alpha \beta$-blockers) have been shown to prevent the occurrence of cardiovascular disease. ${ }^{114,226}$ For each class of the drug, there are compelling indications, contraindications and conditions that require the careful use of drugs. When these conditions are present, condition-matched antihypertensive drugs should be selected (Tables 5-1 and 5-2).

In hypertensive patients without specific conditions, the antihypertensive drug to be first administered should be selected from $\mathrm{Ca}$ channel blockers, ARBs, ACE inhibitors and diuretics.

There is evidence that $\beta$-blockers alone or in combination with other drugs are less useful than other drugs with respect to diabetes mellitus-inducing actions $s^{403,404}$ and preventive effects on organ damage/cardiovascular disease. ${ }^{405-409}$ These are primarily based on the results of conventional $\beta$-blockers represented by atenolol. ${ }^{410}$ It is controversial whether there is a difference in the efficacy between this drug and a new class of $\beta$-blocker such as carvedilol. ${ }^{411}$ A conclusion regarding this issue has not been reached.

Diuretics are effective for salt-sensitive hypertension, including hypertension in the elderly. ${ }^{412}$ There is evidence on their preventive effects on stroke in the Japanese. ${ }^{256,413-415}$ These drugs are also appropriate for combination therapy with an ARB or an ACE inhibitor. In the COPE Study, diuretics were significantly more useful than $\beta$-blockers for combination therapy with a Ca channel blocker. ${ }^{415}$ The use of low-dose thiazide diuretics and their analogs inhibits the appearance of metabolic adverse effects.

Therefore, Ca channel blockers, ARBs, ACE inhibitors or low-dose diuretics should be commonly selected as a first-choice drug in hypertensive patients.

2) Use of antihypertensive drugs

The ultimate objective of antihypertensive treatment is to prevent cardiovascular disease. Once antihypertensive drug therapy has been started, the realization of the target control level should always be borne in mind. However, the reality is unsatisfactory, as various investigations have indicated that the target is achieved in only $\sim 50 \%$ of those taking antihypertensive medication. ${ }^{416}$ The frequency 
Table 5-1 Conditions for which major antihypertensive drugs are indicated

\begin{tabular}{|c|c|c|c|c|}
\hline & $\begin{array}{c}\text { Ca channel } \\
\text { blockers }\end{array}$ & $\begin{array}{c}\text { ARBs/ACE } \\
\text { inhibitors }\end{array}$ & $\begin{array}{l}\text { Thiazide } \\
\text { diuretics }\end{array}$ & $\beta$-Blockers \\
\hline \multicolumn{4}{|l|}{ Left ventricular hypertrophy } & \\
\hline Tachycardia & $\begin{array}{l}\text { (Non-dihydro- } \\
\text { pyridines) }\end{array}$ & & & ○ \\
\hline \multicolumn{5}{|l|}{ Angina pectoris } \\
\hline \multicolumn{5}{|l|}{ Post myocardial infarction } \\
\hline \multicolumn{5}{|l|}{$C K D$} \\
\hline \multicolumn{5}{|l|}{ Proteinuria (-) } \\
\hline \multicolumn{5}{|l|}{ Proteinuria (+) } \\
\hline \multicolumn{5}{|l|}{$\begin{array}{l}\text { Chronic phase of } \\
\text { cerebrovascular disorders }\end{array}$} \\
\hline \multicolumn{5}{|l|}{ Diabetes mellitus/MetS ${ }^{c}$} \\
\hline \multicolumn{5}{|l|}{ Osteoporosis } \\
\hline Aspiration pneumonia & & $\begin{array}{c}\text { (ACE } \\
\text { inhibitors) }\end{array}$ & & \\
\hline
\end{tabular}

Abbreviations: ACE, angiotensin-converting enzyme; ARB, angiotensin-receptor blockers; MetS, metabolic syndrome.

aAdministration should be started at a low dose, and the dose should be gradually increased carefully.

${ }^{\mathrm{b}}$ Caution is needed in patients with coronary spastic angina pectoris.

${ }^{\mathrm{C} M e t a b o l i c ~ s y n d r o m e . ~}$

Table 5-2 Contraindications for major antihypertensive drugs and conditions requiring careful administration

\begin{tabular}{|c|c|c|}
\hline & Contraindications & Conditions that require careful use \\
\hline Ca channel blockers & $\begin{array}{l}\text { Bradycardia } \\
\text { (non-dihydropyridines) }\end{array}$ & Heart failure \\
\hline ARB & $\begin{array}{l}\text { Pregnancy } \\
\text { Hyperkalemia }\end{array}$ & Renal artery stenosis ${ }^{a}$ \\
\hline ACE inhibitors & $\begin{array}{l}\text { Pregnancy } \\
\text { Angioneurotic edema } \\
\text { Hyperkalemia }\end{array}$ & Renal artery stenosis ${ }^{a}$ \\
\hline $\begin{array}{l}\text { Diuretics } \\
\text { (thiazide) }\end{array}$ & Hypokalemia & $\begin{array}{l}\text { Gout } \\
\text { Pregnancy } \\
\text { Impaired glucose tolerance }\end{array}$ \\
\hline$\beta$-Blockers & $\begin{array}{l}\text { Asthma } \\
\text { Marked bradycardia }\end{array}$ & $\begin{array}{l}\text { Impaired glucose tolerance } \\
\text { Obstructive pulmonary disease } \\
\text { Peripheral artery disease }\end{array}$ \\
\hline
\end{tabular}

Abbreviations: ACE, angiotensin-converting enzyme; ARB, angiotensin-receptor blockers. ${ }^{a} \mathrm{As}$ a rule, ARBs/ACE inhibitors are contraindicated for patients with bilateral renal artery stenosis.

with which the target control level can be achieved using a single drug is low. ${ }^{417}$ The use of antihypertensive drugs to achieve the target level of blood pressure control is shown in Figure 5-1. Antihypertensive drug therapy should be started with a single drug at a low dose. If adverse effects appear or little hypotensive effect is noted, the drug should be replaced by another class of drug. If the hypotensive effect is still insufficient, the dose should be increased or a different class of antihypertensive drug at a low dose should be used concomitantly. In this case, combination therapy with a different class of antihypertensive drug at a low dose shows more marked hypotensive effects compared with doubling the dose of the antihypertensive drug. ${ }^{418,419}$ In patients with grade II or severer hypertension ( $\geqslant 160 / 100 \mathrm{~mm} \mathrm{Hg})$, antihypertensive medication may be started with a single drug at a standard dose or with a combination of two drugs at low doses. ${ }^{113,260}$ However, fixed-combination antihypertensive drugs as a first-choice drugs are not covered by health insurance. An increase in the dose of

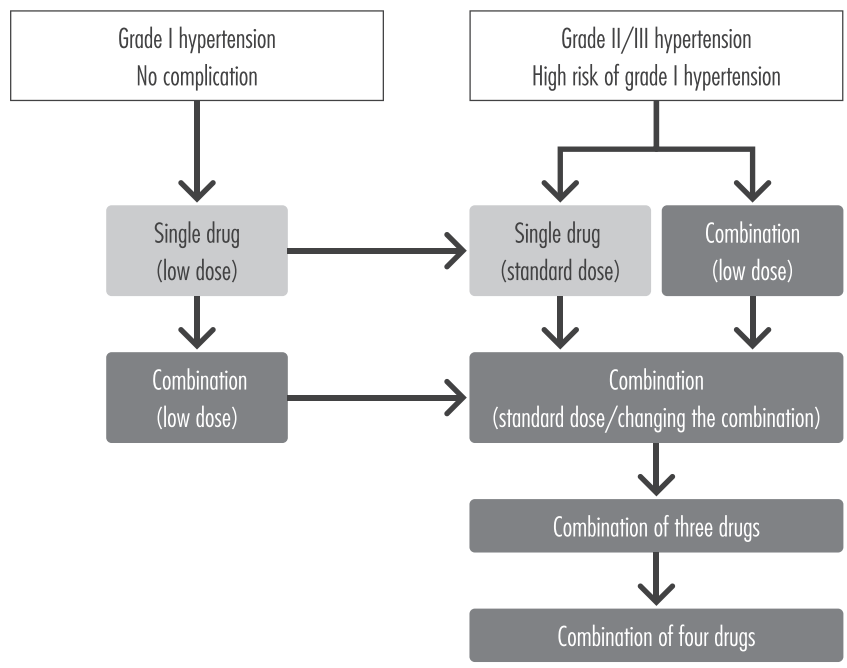

Figure 5-1 Use of antihypertensive drugs to achieve the target level of blood pressure control. A full color version of this figure is available at the Hypertension Research journal online.

antihypertensive drugs other than ACE inhibitors and ARBs increases the frequency of adverse effects. ${ }^{420}$ If the target control level cannot be achieved by combination therapy with two drugs, a combination of three drugs should be introduced. If necessary, four drugs may be used in combination.

The procedures of antihypertensive therapy for hypertension in the absence of compelling indications are shown in Figure 5-2. If a condition for which antihypertensive therapy should be indicated is present, a single, condition-matched antihypertensive drug or combination therapy with other classes of antihypertensive drug may be considered. To facilitate long-term adherence, antihypertensive drugs effective with once-a-day administration are desirable. Many clinical studies have suggested the importance of 24-h blood pressure control by also paying attention to the out-of-clinic blood pressure. The effects of many antihypertensive drugs commercially available today do not persist for $24 \mathrm{~h}$ if used clinically. If the trough blood pressure measured at home or over $24 \mathrm{~h}$ is high, the time of administration may be tentatively changed from morning to evening, the dose split into morning and evening or an additional dose taken in the evening or before going to bed. ${ }^{421-423}$

A gradual rate of blood pressure reduction that achieves the target level in a few months is desirable, because it causes fewer adverse effects. In particular, in elderly patients in whom the ability to regulate blood pressure is reduced, a rapid decrease should be avoided. However, with patients at a high risk of cardiovascular disease, there are results indicating that the difference in the rate of blood pressure reduction during the first 1-3 months after commencing treatment affected the subsequent occurrence of disease; ${ }^{424}$ therefore, in these cases, the attainment of the target level within several weeks is recommended.

\section{3) Drug interactions}

Interactions between antihypertensive drugs may enhance the hypotensive effect or offset adverse effects in some combinations, but may aggravate adverse effects in others. ${ }^{425}$ Particular attention is necessary with regard to the enhancement of the cardioinhibitory effect by a combination of a $\beta$-blocker and a non-dihydropyridine (non-DHP) Ca channel blocker, aggravation of hyperkalemia by a combination of an RA system inhibitor and a potassium-sparing diuretic and an increase in the frequency of withdrawal syndrome by a combination of 
a central sympatholytic drug and a $\beta$-blocker. Interactions between antihypertensive drugs and drugs for the treatment of other diseases include the attenuation of the hypotensive effects of diuretics, $\beta$ blockers, ACE inhibitors and ARBs by nonsteroidal anti-inflammatory drugs, enhancement of the hypotensive effects of Ca channel blockers and $\beta$-blockers by histamine $\mathrm{H} 2$-receptor blockers, an increase in the blood digoxin concentration by a combination of digoxin and a nonDHP Ca channel blocker, and interactions between DHP Ca channel blockers and antifungal/antimicrobial drugs (see the end of this volume). The concomitant use of an ARB or an ACE inhibitor with a nonsteroidal anti-inflammatory drug or a diuretic may cause acute renal insufficiency or an excessive decrease in blood pressure, particularly in elderly patients, with unfavorable water intake-/vomiting-/ diarrhea-/excessive sweating-related dehydration or under restriction of salt intake. A well-known example of food-drug interaction is an increase in the blood concentration of DHP Ca channel blockers after their administration following the consumption of grapefruit or grapefruit juice.

Some patients taking sympathetic drugs, antidepressants, anesthetics or antitumor drugs that increase blood pressure as an adverse effect (see Section 7 of Chapter 13, DRUG-INDUCED HYPERTENSION) require treatment with antihypertensive drugs.

\section{4) Dose reduction and withdrawal of antihypertensive drugs}

Blood pressure shows seasonal fluctuations, and a temporary decrease in the dose or withdrawal may be considered in patients who show a decrease in blood pressure in summer. Conversely, because of the increase in blood pressure in winter, dose elevation or the readministration of the antihypertensive drug becomes necessary in many patients. Even if a normal blood pressure has been maintained for 1 year or more by antihypertensive medication, blood pressure often increases to a hypertensive level usually within 6 months of a reduction in dose or withdrawal of the drug. The percentage of patients in whom blood pressure could be maintained after the withdrawal of antihypertensive medication varies widely among

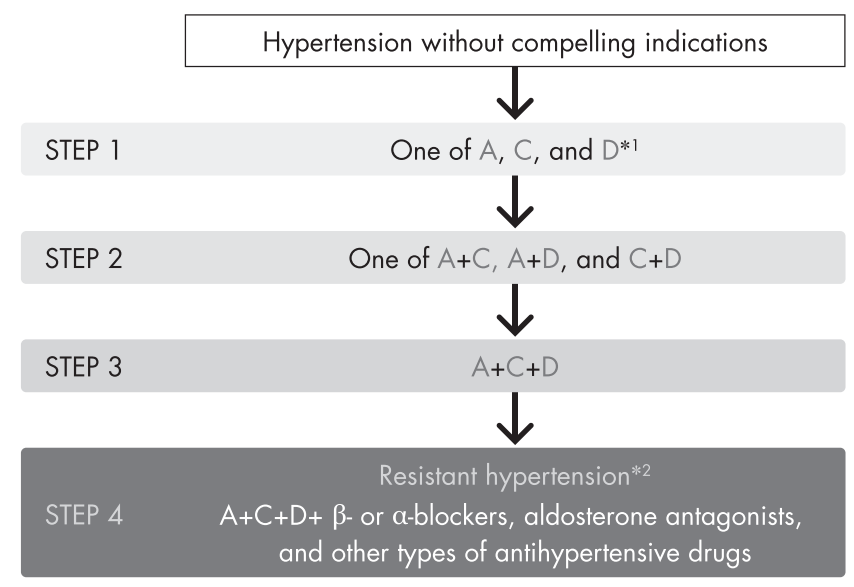

First-choice drugs: A: ARBs, ACE inhibitors, C: Ca channel blockers, $D$ : Thiazide diuretics, thiazide analogues,

Figure 5-2 Procedures of hypertension treatment in the absence of compelling indications. ${ }^{* 1}$ In elderly patients, administration should be started at $1 / 2$ of the standard dose, and the dose should be increased at 1-3-month intervals. ${ }^{* 2}$ See the section 5 'Strategies for resistant or poorly controlled hypertension'. A full color version of this figure is available at the Hypertension Research journal online. studies from 3 to $74 \%$. The characteristics of patients in whom a normal blood pressure could be maintained even after withdrawal include having grade I hypertension before treatment, a young age, normal body weight, low salt intake, being a nondrinker, using only one antihypertensive drug and having no organ damage. ${ }^{426}$ Therefore, withdrawal of antihypertensive medication may be attempted exclusively in patients with grade I hypertension without organ damage or complications on the condition that an appropriate lifestyle is maintained and blood pressure is monitored periodically. However, it cannot be recommended for other hypertensive patients.

\section{COMBINATION THERAPY}

To achieve the target level of blood pressure control, combination therapy with two or three drugs is performed in many patients. A meta-analysis showed that the hypotensive effects of a combination of different classes of antihypertensive drug were more marked than those of the double-dose administration of the same drug. ${ }^{418}$ Evidence that strict management of blood pressure by combination therapy contributes to the further prevention of cardiovascular events based on large-scale clinical studies is being accumulated. The usefulness of combinations of drugs that cancel out each other's adverse effects, such as that of a diuretic and an ACE inhibitor or ARB, is also supported from the point of view of pharmacological actions. Several studies have suggested that combination therapy with an RA system inhibitor and a Ca channel blocker ${ }^{427}$ and that with an RA system inhibitor and a diuretic ${ }^{428}$ are more useful than that with a $\beta$-blocker and a diuretic. Currently, the following combinations are recommended among first-choice drugs: (1) an ACE inhibitor or ARB + a Ca channel blocker, (2) an ACE inhibitor or ARB + a diuretic and (3) a Ca channel blocker + a diuretic (Figure 5-3).

\section{1) Merits of combination therapy}

In the RENAAL Study, ${ }^{429}$ combination therapy with a Ca channel blocker and an ARB inhibited progression to end-stage renal disease. In Japan, several studies have also demonstrated that the enhancement of hypotensive effects and reduction of proteinuria by combination therapy with an ACE inhibitor or ARB at a standard dose and a Ca channel blocker are more marked than those by high-dose ARB therapy. ${ }^{430-432}$ In the OSCAR Study, ${ }^{433}$ in which high-dose ARB therapy was compared with standard-dose ARB + Ca channel blocker therapy in elderly, high-risk Japanese patients, the hypotensive effects in the combination therapy group were more marked than in the

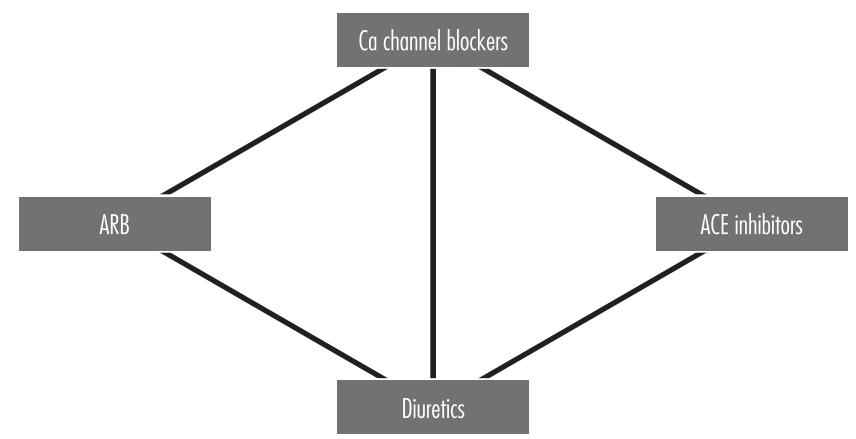

Figure 5-3 Combination of two drugs. ${ }^{*}$ Combination therapy with an ARB and an ACE inhibitor is not commonly used. If the two drugs are concomitantly used to protect the kidney, they must be carefully administered while monitoring kidney function and considering the risk of hyperkalemia. A full color version of this figure is available at the Hypertension Research journal online. 
high-dose group. Among patients with a history of cardiovascular disease, the incidence of events was significantly lower in the former. On the other hand, in the HIJ-CREATE Study, ${ }^{434}$ when selecting a Ca channel blocker as a base in hypertensive patients with cardiovascular disease, the incidence of cardiovascular events in the ARB-combined group was lower than in the non-ARB-combined group. In the ADVANCE Study, ${ }^{435}$ the incidence of diabetic complications, that of coronary events, cardiovascular mortality rate and total mortality rate in diabetics taking a combination of an ACE inhibitor and a diuretic in addition to current antihypertensive treatment were lower than in the placebo group. In the HYVET Study, ${ }^{244}$ a decrease in blood pressure in the ACE inhibitor + diuretic therapy group consisting of elderly patients aged over 80 years was more marked than in the placebo group, and the risk of stroke, mortality rate and total mortality rate were significantly lower in the former. The PROGRESS Study, ${ }^{256}$ which was conducted as a recurrent stroke inhibition study, showed the preventive effects of combination therapy with an ACE inhibitor and a diuretic on recurrent stroke. Thus, a large number of clinical studies have indicated that positive antihypertensive treatment with combination regimens decreases the number of events.

2) Combinations of antihypertensive drugs in combination therapy In the ASCOT Study, ${ }^{427}$ a combination of an ACE inhibitor and a Ca channel blocker exhibited more potent hypotensive effects than that of a $\beta$-blocker and a diuretic, and prevented cardiovascular events. In the GUARED Study, ${ }^{436}$ in which the effects of combination therapy with an ACE inhibitor and a diuretic were compared with those of that with an ACE inhibitor and a Ca channel blocker in type 2 diabetics with albuminuria, the former more markedly decreased urinary albumin excretion than the latter. However, a decrease in the eGFR in patients undergoing the latter was less marked than in those undergoing the former. In a large-scale, randomized, double-blind study (ACCOMPLISH), ${ }^{437}$ in which the effects of similar combinations of antihypertensive drugs were investigated, combination therapy with an ACE inhibitor and a Ca channel blocker prevented the occurrence of cardiovascular events and death in comparison with that with an ACE inhibitor and a diuretic. A subanalysis ${ }^{438}$ showed that the urinary albumin-decreasing effects of combination therapy with an ACE inhibitor and a Ca channel blocker were less marked than those of that with an ACE inhibitor and a diuretic, but the preventive effects of the former on the progression of CKD were more potent than those of the latter. On the basis of these results, in the Guide for CKD Treatment, ${ }^{439}$ it is recommended that an ACE inhibitor or ARB should be combined with a Ca channel blocker in high-risk patients regarding cardiovascular events and with a diuretic in those with fluid retention. Furthermore, a subanalysis of the ACCOMPLISH Study with respect to the BMI level ${ }^{440}$ indicated that combination therapy with an ACE inhibitor and a Ca channel blocker prevented cardiovascular events regardless of the BMI level, whereas there were no preventive effects of combination therapy with an ACE inhibitor and a diuretic in any group other than the obesity group. A study to compare the preventive effects of combination therapy with an ARB and a diuretic on cardiovascular events with those of that with an ARB and a Ca channel blocker, establishing the target level of blood pressure control as $<140 / 90 \mathrm{~mm} \mathrm{Hg}$, is being conducted.

In the COPE Study in Japan, ${ }^{415}$ the results were compared among three combinations: a Ca channel blocker, as a base, + a diuretic; a Ca channel blocker + an ARB; and a Ca channel blocker + a $\beta$-blocker. There were no significant differences in the incidence of complicated cerebro-/cardiovascular events among the three groups. However, both the incidence of stroke, excluding cardiovascular death, myocardial infarction and TIA, and that of stroke including TIA in the diureticcombined group were lower than in the $\beta$-blocker-combined group. The incidence of new-onset diabetes mellitus in the ARB-combined group was lower than that in the $\beta$-blocker-combined group. In the VALUE Study involving high-risk hypertensive patients, ${ }^{424}$ the preventive effects of combination therapy with a Ca channel blocker and a diuretic on complicated cardiovascular events were similar to those of that with an ARB and a diuretic. According to other studies, a combination of a $\mathrm{Ca}$ channel blocker and a diuretic exhibited preventive effects on the occurrence of cardiovascular events, including stroke, and the effects were more marked than its hypotensive effects. ${ }^{274,441}$ In the INVEST Study, ${ }^{442}$ a non-DHP Ca channel blocker was used in patients with coronary artery disease, and the preventive effects of combination therapy with a Ca channel blocker and an ACE inhibitor on total mortality, nonfatal myocardial infarction and nonfatal stroke were similar to those of that with a $\beta$-blocker and a diuretic.

A meta-analysis showed that combination therapy with an ACE inhibitor and an ARB more markedly decreased the urinary protein level than monotherapy. ${ }^{43}$ However, the ONTARGET Study ${ }^{444,445}$ indicated that, although the urinary protein level in the ACE inhibitor + ARB group was lower than in the monotherapy group, the results regarding the introduction of dialysis, an increase in Cr to twofold, and mortality were less favorable in the former. Generally, this combination is not recommended. When selecting this combination, treatment should be started at a low dose, and careful follow-up is needed.

A combination of a $\beta$-blocker and a Ca channel blocker was used in many patients with coronary artery disease in the ACTION Study. In particular, it was useful for preventing events in hypertensive patients. ${ }^{446}$

Aldosterone antagonists are often combined with Ca channel blockers, diuretics or $\beta$-blockers. There is evidence regarding improvement in the prognosis of heart failure, as demonstrated for ACE inhibitors, ARBs and $\beta$-blockers. In the Guidelines for Heart Failure Treatment, the administration of aldosterone antagonists is also recommended. ${ }^{44-449}$ Therefore, an ACE inhibitor or ARB should be combined with a $\beta$-blocker, diuretic or aldosterone antagonist in patients with heart failure.

When combining an ACE inhibitor or ARB with an aldosterone antagonist or potassium-sparing diuretic, the kidney function and serum $\mathrm{K}$ level must be monitored.

If the hypotensive effects of two-drug therapy are not sufficient, combination therapy with three drugs, that is, an ACE inhibitor/ARB, a Ca channel blocker and a diuretic, should be performed. If the target blood pressure is not reached, the additional administration of the following drugs must be considered: (1) $\beta$-blockers, (2) $\alpha$-blockers, (3) aldosterone antagonists, (4) renin inhibitors and (5) others (nonDHP Ca channel blockers, central sympatholytic drugs or hydralazine) (see Section 5 of Chapter 5, STRATEGIES FOR RESISTANT HYPERTENSION AND POORLY CONTROLLED HYPERTENSION).

\section{FIXED-COMBINATION DRUGS}

A reduction in the number of tablets to be taken and simplification of the prescription through the use of fixed-combination drugs is advantageous for improving adherence. ${ }^{317,450}$ The ADVANCE Study, ${ }^{435}$ which compared the effects of a fixed-combination drug of an ACE inhibitor and a diuretic with those of a placebo in diabetics, indicated the usefulness of the fixed-combination drug. Adherence was similar between the fixed-combination drug and placebo. A metaanalysis showed that fixed-combination drugs exhibited more potent hypotensive effects than combination therapy with respective drugs by 
improving adherence, and that these drugs improved the rate at which the target level of blood pressure control is achieved. ${ }^{450}$ On the other hand, the dose of a fixed-combination drug is fixed, and initial administration may cause an excessive decrease in blood pressure. Therefore, initially, a single drug or a combination of two drugs should be administered, and, after establishing the dose/doses, it should be switched to a fixed-combination drug.

In Japan, fixed-combination drugs of an ARB and a diuretic and those of an ARB and a Ca channel blocker are currently available. The price of a fixed-combination drug is less expensive than the total price of respective drugs, and there is a medicoeconomical merit. As the efficacy and safety of fixed-combination drugs are confirmed, these drugs may be used more frequently.

\section{CHARACTERISTICS AND MAJOR ADVERSE EFFECTS OF VARIOUS ANTIHYPERTENSIVE DRUGS}

\section{1) Ca channel blockers}

Ca channel blockers produce hypotensive effects by inhibiting the L-type voltage-dependent Ca channel involved in the influx of extracellular $\mathrm{Ca}$ ions, thus relaxing the vascular smooth muscle and reducing peripheral vascular resistance. In Japan, Ca channel blockers such as DHPs and benzothiazepines are used as antihypertensive drugs. The former is primarily selected. Their primary pharmacological actions are as follows: (1) coronary and peripheral vasodilation, (2) suppression of the cardiac contractile force and (3) suppression of the conduction system. DHPs rapidly and potently reduce blood pressure and show little cardioinhibitory effect at clinical doses. Short-acting preparations rather induce tachycardia because of a reflex increase in the sympathetic tone. Non-DHP Ca channel blockers have slower and milder hypotensive effects accompanied by a cardioinhibitory effect. DHP Ca channel blockers exhibit potent vasodilative actions and the most potent hypotensive effects among the antihypertensive drugs currently available. They have also been indicated to show slight Na diuretic actions through an increase in the renal blood flow/glomerular filtration rate and a reduction in aldosterone secretion. ${ }^{451,452}$ As organ blood flow is maintained, they are positively indicated for patients with organ damage or elderly patients, and used as a first-choice drug in many patients. Many DHPs are administered once a day. Amlodipine has the longest half-life in the circulation, and sustained-release nifedipine tablets have a novel pharmaceutical design, with a consequent long duration of action and milder adverse effects, such as a reflex sympathomimetic action. It exerts no adverse effect on glucose, lipid or electrolyte metabolism. It has also been reported to induce the regression of left ventricular hypertrophy and delay the progression of atherosclerotic plaques. ${ }^{453,454}$ Some Ca channel blockers, such as cilnidipine, efonidipine, benidipine and azelnidipine, which inhibit N-type or T-type Ca channels and have sympatholytic actions, have been reported to show marked antiproteinuric effects in hypertensive patients with kidney disease. ${ }^{430,455-457}$ Antihypertensive therapy with $\mathrm{Ca}$ channel blockers exhibits central blood pressure-decreasing effects, ${ }^{458,459}$ which cannot be detected based on brachial blood pressure, and blood pressure variabilityreducing effects. ${ }^{460,461}$ These effects are advantageous from the viewpoint of the quality of blood pressure control, and are evaluated as characterizing Ca channel blockers. The adverse effects of Ca channel blockers include palpitations, headache, hot flushes, edema, gingival growth and constipation. Non-DHP Ca channel blockers must not be used in patients with heart failure or marked bradycardia because of their cardioinhibitory actions, and sufficient caution is necessary regarding their use in elderly patients with latent cardiac disorders or their concomitant use with digitalis or $\beta$-blockers.

\section{2) ARBs}

ARBs are the second most common antihypertensive drugs in Japan, following Ca channel blockers. They produce a hypotensive effect by specifically binding to angiotensin II (AII) type 1 (AT1) receptors and inhibiting strong AII-mediated vasoconstriction, body fluid retention and sympathetic activity. On the other hand, at the tissue level, nonACE-mediated AII production systems, such as the chymase system, are present. ARBs also inhibit the actions of AII at the receptor level. The administration of ARBs may increase the blood AII level through a feedback mechanism and stimulate AII type 2 receptors, which antagonize the cardiovascular actions of AT1 receptors. ${ }^{462}$ AII/AT1 receptors increase blood pressure and directly induce organ damage; therefore, inhibition of AII/AT1 receptors may prevent organ damage and disease onset in addition to hypotensive effects. ARBs are used alone or in combination with Ca channel blockers or diuretics and for the treatment of grades I-III hypertension. The cardioprotective effects of ARBs are that they inhibit cardiac hypertrophy and improve the outcome of heart failure. The preventive effects of telmisartan and ramipril on myocardial infarction were similar in the ONTARGET Study, ${ }^{444}$ but a meta-analysis suggested that the effects of ARBs are less marked than those of ACE inhibitors. ${ }^{402}$ In the kidneys, ARBs reduce the intraglomerular pressure by dilating efferent arterioles. In addition, they prevent exacerbation of the renal function in the long term by decreasing the urinary protein level and inhibiting glomerular sclerosis/ interstitial fibrosis. ${ }^{429,463,464}$ They have also been reported to improve the regulation of cerebral blood flow and prevent atherosclerosis. 424,465 In addition, they improve insulin sensitivity and prevent the new occurrence of diabetes mellitus. ${ }^{466,467}$ For these reasons, ARBs are used as the first choice for patients with complications of the heart, kidney to brain and those with diabetes mellitus. Subanalyses of clinical studies involving patients with heart failure have shown that ARBs prevent the occurrence of atrial fibrillation, but a large-scale clinical study reported negative results regarding their preventive effects on the occurrence of atrial fibrillation when patients are not limited to those with heart failure. ${ }^{468-470}$ The combination with a diuretic is advantageous not only because of the synergism of hypotensive effects but also because it offsets adverse effects on electrolyte and glucose metabolism. Combination therapy with a Ca channel blocker enhances hypotensive effects and preventive effects on the onset of cardiovascular disease, and reduces $\mathrm{Ca}$ channel blocker-related edema.

In Japan, seven types of ARBs are commercially available. However, there are slight differences among these types. In particular, some ARBs reduce the blood uric acid level through a uric acid transporter ${ }^{471}$ and stimulate peroxisome proliferator-activated receptor- $\gamma$ ), which is involved in the improvement of glucose metabolism. ${ }^{472}$ Evidence regarding the uric acid-reducing effects of losartan is being accumulated. ${ }^{473-476}$ In the Guidelines for Gout published by the American College of Rheumatology, ${ }^{477}$ losartan is described as a drug with uric acid-excreting actions, although it is stated that its uric acid-excreting actions are not approved. Small-scale clinical studies have indicated that telmisartan and irbesartan, which activate peroxisome proliferator-activated receptor- $\gamma$, improve insulin resistance and lipid metabolism, ${ }^{478-481}$ but their clinical significance remains to be clarified.

The adverse effects are infrequent regardless of the dose. ${ }^{420}$ However, administration to pregnant or breast-feeding women is contraindicated, and ARBs should be carefully administered to patients with severe liver dysfunction. ARBs must not be used in patients with bilateral renal artery stenosis or those with one kidney and unilateral renal artery stenosis, in principle, because of the risk of a rapid reduction in renal function. A decrease in body fluid volume and $\mathrm{Na}$ 
deficiency are also quasi-contraindications. In patients with $\mathrm{CKD}$, renal function may deteriorate, and the eGFR and serum $\mathrm{K}$ level should be measured within 2 weeks to 1 month after the start of administration. Subsequent monitoring is also necessary. If the eGFR decreases by $30 \%$, or more, of the pretreatment value, or if the serum $\mathrm{K}$ level increases to $5.5 \mathrm{mEql}^{-1}$ or more, the dose of the drug should be decreased, or administration must be discontinued, and the physician should consult a doctor who specializes in diagnosing and treating kidney disease or hypertension. ${ }^{439}$ If the eGFR is $30 \mathrm{ml} \mathrm{min}^{-1}$ per $1.73 \mathrm{~m}^{2}$ or less, administration should be carefully started at a low dose, and considerations such as dose reduction are necessary, because renal function may rapidly deteriorate after the start of administration. ${ }^{439}$ Attention to hyperkalemia is necessary while using ARBs with a potassiumsparing diuretic. In 2010, an article suggesting that ARBs increase the risk of carcinogenesis was published, but a meta-analysis conducted by the Food and Drug Administration in the United States ruled out the association between ARBs and an increase in the risk of carcinogenesis (http://www.fda.gov/Drugs/DrugSafety/ucm257516.htm).

\section{3) ACE inhibitors}

ACE inhibitors inhibit the RA system, which is a strong pressor system, in blood and tissue, and simultaneously stimulate the kallikrein-kinin-prostaglandin system, which is a depressor system. This may also be involved in hypotensive effects.

The BPLTTC meta-analysis ${ }^{402}$ showed that ACE inhibitors significantly reduced the risk of coronary artery disease. ACE inhibitors reportedly increase the production of plasminogen/activator (t-PA). Activation of the fibrinolytic system may be involved in this. ${ }^{482}$ Many large-scale clinical studies have also indicated that ACE inhibitors decrease the incidence of cardiovascular complications following myocardial infarction, improving the prognosis; these drugs are useful for the secondary prevention of myocardial infarction. In the Guidelines for the Secondary Prevention of Myocardial Infarction published by the Japanese Circulation Society, it is described that an ACE inhibitor should be selected as a first-choice RA system inhibitor for the secondary prevention of myocardial infarction, and that ARBs may be used only when there is intolerance to ACE inhibitors. ${ }^{483}$ The hypotensive effect of an ACE inhibitor is nearly the same as, or slightly weaker than, that of an ARB. The most frequent adverse effect is dry cough due to the enhancement of bradykinin activity, which is observed in $20-30 \%$ of patients within 1 week to several months after commencing administration, but it is quickly resolved by the discontinuation of treatment. Dry cough is frequent in East Asians, including Japanese. ${ }^{484}$ For this reason, the maximum doses of ACE inhibitors in Japan were established as lower than in Europe and the United States. This also contributes to their hypotensive effects. The induction of a cough has also been suggested to prevent aspiration pneumonia in elderly patients taking ACE inhibitors. ${ }^{485}$ As an important adverse effect, angioneurotic edema occurs infrequently. A study reported that combination therapy with a DPP4 inhibitor, a drug for type 2 diabetes mellitus, which has recently been used in a rapidly increasing number of patients, increased the incidence of angioneurotic edema. ${ }^{486}$ When angioneurotic edema occurs, dyspnea may lead to a serious condition; therefore, administration should be immediately discontinued, and appropriate treatment must be performed. As shock or anaphylactoid symptoms may occur, an ACE inhibitor is contraindicated for patients undergoing apheresis with an adsorber consisting of dextran sulfate-fixed cellulose, tryptophan-fixed polyvinyl alcohol or polyethylene-telephthalate and those receiving hemodialysis with acrylonitrile-sodium methallylsulfonate membrane. As many drugs are excreted through the kidney, their administration should be started at a low dose in patients with kidney damage. Other adverse effects and cautions are the same as those of ARBs.

\section{4) Direct renin inhibitors}

Among direct renin inhibitors, only aliskiren is currently available in Japan. Hypertension is an indication to be covered by health insurance. Direct renin inhibitors belong to RA system inhibitors in a wide sense. Their inhibitory actions on the RA system are common with those of ARBs and ACE inhibitors. However, they inhibit renin enzyme activity, differing from ARBs and ACE inhibitors, and PRA decreases. The half-life of aliskiren is long $(40 \mathrm{~h})$, and its tissue transfer is favorable. This drug exhibits stable hypotensive effects for many hours when administered once a day. The tolerance is also favorable. ${ }^{487,488}$ Aliskiren is particularly indicated when neither an ARB nor ACE inhibitor can be used because of the adverse effects despite a condition for which an RA system inhibitor should be positively indicated.

A study reported that combination therapy with an RA system inhibitor enhanced the proteinuria-reducing effects. ${ }^{489}$ However, in the ALTITUDE Study, in which the efficacy of combination therapy with aliskiren was compared with that of conventional treatment involving RA system inhibitors in high-risk patients with type 2 diabetes mellitus, there was no further decrease in the incidence of complicated cardiovascular/renal events, and the incidences of hyperkalemia and hypotension increased. ${ }^{490}$ Therefore, combination therapy with a direct renin inhibitor and another RA system inhibitor (ARBs and ACE inhibitor) is not recommended for hypertensive patients with diabetes, those with CKD and an eGFR of $<60 \mathrm{mlmin}^{-1}$ per $1.73 \mathrm{~m}^{2}$, and those with heart failure and a reduction in contractility.

Serious adverse effects include vascular edema, anaphylaxis, hyperkalemia and kidney dysfunction. Combination therapy with itraconazole or cyclosporine is contraindicated. In addition, as a rule, administration to patients with bilateral renal artery stenosis and pregnant women is also contraindicated, as described for other RA system inhibitors (ARBs and ACE inhibitors).

\section{5) Diuretics}

The Japanese are still characterized by a high salt intake, and saltsensitive hypertension is frequent. In antihypertensive treatment, salt reduction is important, but a diuretic at a low dose may be used in hypertensive patients in whom salt restriction is difficult. In large-scale clinical studies, the proportion of patients taking a diuretic in addition to other classes of antihypertensive drugs was also high, and even diuretic therapy alone prevented cardiovascular events. ${ }^{237,246}$ Furthermore, diuretics are inexpensive. Diuretics include thiazide-type diuretics (thiazide diuretics and thiazide-like diuretics), loop diuretics and potassium-sparing diuretics. With respect to potassium-sparing diuretics, see Section 3 of Chapter 5, Article 8, Aldosterone antagonists and potassium-sparing diuretics.

(1) Mechanism of hypotensive action. As antihypertensive drugs, thiazide-type diuretics are commonly used. With respect to kidney function, they are selected in patients with an eGFR of $30 \mathrm{ml} \mathrm{min}^{-1}$ per $1.73 \mathrm{~m}^{2}$ or more. They decrease the circulating blood volume by inhibiting $\mathrm{Na}$ reabsorption in the renal distal tubules, but exhibit hypotensive effects by reducing peripheral vascular resistance in the long term. Drugs with a thiazide structure are classified as thiazide diuretics, and thiazide structure-free drugs are characterized as thiazide-like diuretics. In the JSH2014 Guidelines, the two types of drugs are regarded as thiazide-type diuretics. It is controversial whether the 
clinical efficacy differs between thiazide diuretics and thiazide-like diuretics. However, evidence is limited, and there is no large-scale RCT directly comparing the two types of drug. Therefore, in the JSH2014 Guidelines, both types are not distinguished. On the other hand, loop diuretics are initially administered to patients with an eGFR of $<30 \mathrm{ml} \mathrm{min}^{-1}$ per $1.73 \mathrm{~m}^{2}$. Loop diuretics exhibit diuretic actions by inhibiting $\mathrm{NaCl}$ reabsorption in the ascending limbs of the loop of Henle. They show more marked diuretic effects but less potent hypotensive effects compared with thiazide-type diuretics. If the efficacy is insufficient, marked diuretic effects may be obtained by combining a loop diuretic with a thiazide-type diuretic.

(2) Indications in terms of the mechanism of hypotensive action. Diuretics may be particularly effective in hypertensive patients with increased salt sensitivity, such as the elderly, patients with low renin hypertension, hypertensive patients with CKD, those with diabetes mellitus and those with insulin resistance. They are also useful for decreasing blood pressure in hypertensive patients in whom salt restriction is difficult, those with an excessive body fluid volume related to edema or patients with resistant hypertension. Furthermore, their preventive effects on heart failure are marked. ${ }^{491}$

(3) Administration method. The administration of thiazide-type diuretics should be started at a low dose (concerning fixed-combination drugs, there is a dose corresponding to $1 / 4$, but, generally, a half dose is used), and thus the appearance of adverse effects can be prevented and favorable hypotensive effects can be obtained. With respect to the appropriate doses of diuretics, see the explanations described at the end of this volume regarding the recommended dose of each antihypertensive drug. When administering diuretics to patients with resistant hypertension, therapy at a dose higher than the recommended dose should be considered while paying attention to the influence on electrolytes and metabolism. Furthermore, hypotensive effects are enhanced by combining a diuretic with other classes of antihypertensive drugs, but combination therapy with $\beta$-blockers should be avoided, because it affects glucose/lipid metabolism. According to several studies, combination therapy with RA system inhibitors markedly reduced proteinuria. ${ }^{436-438}$ However, kidney dysfunction (decrease in eGFR), an excessive decrease in blood pressure in summer related to seasonal blood pressure changes, hyponatremia and hypokalemia must be considered.

(4) Adverse effects. The adverse effects of diuretics include electrolyte abnormalities, such as hyponatremia (hyponatremia related to thiazide-type diuretics is frequently observed in thin women, and it causes anti-diuretic hormone secretion abnormalities in some patients), hypokalemia and hypomagnesemia, and unfavorable effects on the metabolic system, such as impaired glucose tolerance, hyperuricemia and hypertriglyceridemia. To prevent hypokalemia, $\mathrm{K}$ preparations or potassium-sparing diuretics should be concomitantly used, and physicians should instruct patients to consume citrus fruits with a high $\mathrm{K}$ content. As serious adverse effects, photodermatosis and thrombocytopenia are observed, although their incidences are low.

\section{6) $\beta$-Blockers (including $\alpha \beta$-blockers)}

$\beta$-Blockers lower the blood pressure by reducing cardiac output, suppressing renin production and inhibiting central sympathetic activities. Although peripheral vascular resistance increases shortly after the initiation of treatment, it returns to its original level after long-term treatment. Indications for the use of $\beta$-blockers are hypertension in young patients showing sympathetic hyperactivity, angina on effort, chronic heart failure, after myocardial infarction, hypertension complicated by tachycardia, hypertension with a high cardiac output, including that caused by hyperthyroidism, high renin hypertension and aortic dissection. $\beta$-Blockers without intrinsic sympathomimetic actions may prevent recurrent myocardial infarction and improve the prognosis of heart failure. On the other hand, a metaanalysis showed that the preventive effects of $\beta$-blockers on the occurrence of heart disease were similar to those of other antihypertensive drugs, whereas the preventive effects of $\beta$-blockers on the occurrence of stroke in elderly patients were less potent than those of other antihypertensive drugs. ${ }^{492}$ In a large-scale clinical study of highrisk hypertensive patients with multiple risk factors (ASCOT-BPLA), a combination of a $\beta$-blocker and a diuretic was found to be inferior to that of a Ca channel blocker and an ACE inhibitor in preventing the occurrence of cardiovascular disease. ${ }^{427} \beta$-Blockers exert adverse effects on glucose and lipid metabolism when used alone or in combination with diuretics. ${ }^{493,494}$ Therefore, they are not the first choice of treatment in elderly patients or when hypertension is complicated by other diseases such as diabetes mellitus and impaired glucose tolerance. However, some studies have reported that $\alpha \beta$ blockers, which also have a vasodilative, $\alpha$-blocking action, and oilsoluble $\beta$-blockers, particularly carvedilol, specifically showed no metabolic adverse effect on their concomitant use with RA system inhibitors, and that the incidence of diabetes mellitus was lower than in patients taking water-soluble $\beta$-blockers. A clinical study to evaluate the long-term outcome is necessary. ${ }^{411,495}$

Bronchial asthma, grade II or severer AV block, Raynaud's phenomenon and pheochromocytoma (when a $\beta$-blocker is not combined with an $\alpha$-blocker, or other than $\alpha \beta$-blockers) are contraindications for $\beta$-blockers. These drugs must be carefully administered to patients with chronic obstructive pulmonary diseases. $\beta$-Blockers may activate $\alpha 1$ receptors, inducing coronary spasm. Therefore, a $\beta$-blocker should be combined with a $\mathrm{Ca}$ channel blocker in patients with vasospastic angina pectoris. As its sudden discontinuation may induce withdrawal symptoms such as angina pectoris and hypertensive attacks, its dose should be gradually reduced before withdrawal. ${ }^{496}$ Caution is needed in its concomitant use with verapamil or diltiazem, because it is most likely to induce bradycardia and heart failure.

\section{7) $\alpha$-Blockers}

$\alpha$-Blockers selectively block $\alpha_{1}$-receptors on the smooth muscle side of the sympathetic nerve terminal. They do not inhibit suppressive $\alpha_{2}$-receptors on the sympathetic nerve terminal side and rarely cause tachycardia, especially when they are the long-acting type. They are used for blood pressure control before surgery on pheochromocytoma, and are administered before sleep for the treatment of morning hypertension. ${ }^{497}$ As first-dose phenomena, they may cause dizziness, palpitation and syncope due to orthostatic hypotension. Therefore, their administration should be started at a low dose with gradual increases.

\section{8) Aldosterone antagonists and potassium-sparing diuretics}

Aldosterone antagonists such as spironolactone and eplerenone may be particularly effective for low renin hypertension, and are also useful for treating resistant hypertension. ${ }^{498}$

As aldosterone affects the cardiovascular system, aldosterone antagonists have an organ-protecting effect. Many RCTs have indicated that aldosterone antagonists improve the prognosis of heart failure or that after myocardial infarction. These drugs are indicated for these diseases with hypertension. ${ }^{499-501}$ 
Spironolactone and eplerenone have been confirmed to reduce proteinuria. ${ }^{502-504}$ However, caution is needed to combination therapy of the aldosterone antagonists with RA system inhibitor specifically for kidney dysfunction or heart failure. As eplerenone may induce hyperkalemia, it is contraindicated for diabetic nephropathy patients with albuminuria or proteinuria and patients with a creatinine clearance of $<50 \mathrm{ml} \mathrm{min}^{-1}$. Therefore, eplerenone can be administered to patients with a creatinine clearance of $50 \mathrm{ml} \mathrm{min}^{-1}$ or above other than those with diabetic nephropathy to decrease blood pressure and reduce proteinuria. This restriction does not apply to spironolactone, but hyperkalemia must also be considered.

Spironolactone induces adverse effects such as gynecomastia, impotence and menorrhalgia, whereas eprelenone causes few adverse effects.

Triamterene shows similar effects by suppressing the amiloridesensitive epithelial $\mathrm{Na}$ channel independently of aldosterone.

\section{9) Centrally acting sympatholytic drugs}

They inhibit sympathetic activities by stimulating $\alpha_{2}$-receptors in the vasomotor center of the medulla oblongata, thus reducing blood pressure. They cause many adverse effects, such as sleepiness, thirst, malaise and impotence, and are usually used when other drugs are not tolerated or when blood pressure control is difficult despite combination therapy. They may also be administered to patients with renal dysfunction. They are administered before sleep for the treatment of morning hypertension, which alleviates their adverse effects. Methyldopa is used for the treatment of pregnancy-induced hypertension. As dizziness or liver dysfunction may occur, caution is needed. The sudden discontinuation of guanabenz or clonidine administration may induce withdrawal symptoms. As the administration of centrally acting sympatholytic drugs alone causes sodium and water retention, the concomitant use of diuretics is recommended.

\section{0) Classic vasodilators}

Classic vasodilators dilate blood vessels by directly acting on the vascular smooth muscle. Hydralazine is used to treat pregnancyinduced hypertension. As it acts quickly, it can also be used for the treatment of hypertensive emergencies. With regard to adverse effects, anginal attacks may be induced. Other adverse effects are headache, palpitation, tachycardia and edema; fulminant hepatitis has been reported, and hence liver disorder is a contraindication. Systemic lupus erythematosus-like symptoms may appear when classic vasodilators are used continuously.

Minoxidil, which is used as a hair growth stimulant, exhibits potent vasodilative actions. A decrease in blood pressure must be considered.

\section{STRATEGIES FOR RESISTANT HYPERTENSION AND POORLY CONTROLLED HYPERTENSION}

\section{POINT 5B}

1. In patients with resistant hypertension or poorly controlled hypertension, responsible factors such as lifestyle-related factors, including excessive salt consumption, obesity and alcohol consumption, poor adherence, white coat hypertension/white coat phenomenon, the inappropriate selection and doses of antihypertensive drugs, sleep apnea syndrome, secondary hypertension such as primary aldosteronism, kidney dysfunction and an increase in the body fluid volume, stress and attenuation of hypotensive effects related to the use of other drugs should be considered (Recommendation grade: A, Evidence level: IVb).
2. After making an inquiry and communicating with the patient, lifestyle modifications and guidance for drug therapy should be performed. In antihypertensive treatment, multiple drugs differing in the action mechanism, including diuretics, should be combined. A sufficient dose of antihypertensive drugs should be used, and the frequency and time of dosing must be considered (Recommendation grade: B, Evidence level: III).

3. Organ damage may be present, and the proportion of high-risk patients is high. In addition, there is a possibility of secondary hypertension. Therefore, consultation with a hypertension specialist should be sought at an appropriate time (Recommendation grade: B, Evidence level: VI).

\section{1) Definition and prevalence}

In many hypertensive patients, blood pressure is not controlled even with the administration of antihypertensive drugs. Patients in whom blood pressure does not decrease to the target level despite the use of three different antihypertensive drugs are regarded as having resistant hypertension. ${ }^{505}$ Those in whom therapy with four or more antihypertensive drugs decreases blood pressure to the target level may also be regarded as having controlled resistant hypertension. However, in a strict sense, resistant or refractory hypertension refers to a condition in which therapy with three antihypertensive drugs, including a diuretic, at appropriate doses in combination with lifestyle modifications does not decrease blood pressure to the target level. ${ }^{113,506}$ Furthermore, patients who do not meet the definition despite unfavorable blood pressure control with 2-3 antihypertensive drugs should also be regarded as having poorly controlled hypertension. It may be practical to perform strategies similar to those for resistant hypertension. Even in patients with poorly controlled or resistant hypertension, a sufficient decrease in blood pressure may be achieved by correcting factors presented in Table 5-3. However, these patients include a high proportion of those with organ damage and high-risk patients, and the physician should refer the patient to a hypertension specialist at an appropriate time..$^{505,507}$

The incidence of resistant hypertension varies among diagnostic criteria, target blood pressure levels and study populations. It is reported to be $<10 \%$ at general clinics, but may exceed $50 \%$ at outpatient nephrology or hypertension clinics. ${ }^{508}$ According to the NHANES 2003-2008 (Health and Nutrition Survey in the United States), the incidence of resistant hypertension was $12.8 \%$ in hypertensive patients receiving treatment. ${ }^{509}$ In this study, patients with a blood pressure of $140 / 90 \mathrm{~mm} \mathrm{Hg}$ or above despite therapy with three antihypertensive drugs or those taking four or more antihypertensive drugs regardless of blood pressure were defined as having resistant hypertension. In Japan, the J-HOME Study involving clinicians/practitioners showed that home or clinic blood pressure control was unfavorable in $13 \%$ of patients taking three or more antihypertensive drugs. ${ }^{71}$ The incidence of resistant hypertension meeting the strict definition described above may be lower, but the actual number is unclear.

In large-scale clinical studies involving a high percentage of highrisk hypertensive patients, such as the ALLHAT, CONVINCE, LIFE, INSIGHT and VALUE, the proportion of patients in whom blood pressure did not decrease to the target level $(<140 / 90 \mathrm{~mm} \mathrm{Hg})$ was $\sim 30-50 \% .^{237,424,428,510,511}$ In the first three studies, $\sim 40 \%$ of patients were taking three or more antihypertensive drugs. In the CASE-J Study, performed in Japan, the number of drugs used was $\sim 1.5$, and a target blood pressure was achieved in $\sim 60 \%$ of patients; ${ }^{512}$ the 
Table 5-3 Factors and strategies for resistant or poorly controlled hypertension in hypertension treatment

\begin{tabular}{|c|c|}
\hline Factors & Strategies \\
\hline \multicolumn{2}{|c|}{ Problems with blood pressure measurement } \\
\hline $\begin{array}{l}\text { Use of an inappropriately } \\
\text { small cuff }\end{array}$ & $\begin{array}{l}\text { Use of a cuff with a width of } 40 \% \text { of the brachial girth and a } \\
\text { length sufficient to cover at least } 80 \% \text { of the brachial girth }\end{array}$ \\
\hline Pseudohypertension & Attention to marked atherosclerosis \\
\hline $\begin{array}{l}\text { White coat hypertension/ } \\
\text { white coat phenomenon }\end{array}$ & $\begin{array}{l}\text { Confirmation by home and/or ambulatory blood pressure } \\
\text { measurement }\end{array}$ \\
\hline \multirow[t]{6}{*}{ Unfavorable adherence } & Sufficient explanation and education \\
\hline & Changing the drug if adverse effects are observed \\
\hline & $\begin{array}{l}\text { Considering psychological factors if drug maladjustment is } \\
\text { repeated }\end{array}$ \\
\hline & Considering economic problems \\
\hline & $\begin{array}{l}\text { Considering the dosing schedule matched with the } \\
\text { patient's lifestyle }\end{array}$ \\
\hline & Showing the physician's positive and empathetic attitude \\
\hline
\end{tabular}

\section{Lifestyle problems}

Excessive salt intake

Obesity (excessive caloric

intake and lack of exercise)

Excessive alcohol

consumption

Sleep apnea syndrome

Volume overload

Inappropriate use of

diuretics

Progression of nephropathy

Inappropriate combinations/ doses of antihypertensive

drugs

Insufficient duration

of drug efficacy

Drugs/foods that may

increase blood pressure

Secondary hypertension

Explanation of the significance and necessity of salt reduction

Repeated guidance in cooperation with a nutritionist

Repeated guidance in restriction of energy intake and

exercise

Guidance to restrict alcohol intake at $\leqslant 20-30 \mathrm{ml}$ ethanol per day

Appropriate treatments such as continuous positive airway pressure

In combinations of three or more drugs, one should be a diuretic. Selection of a loop diuretic in patients with advanced kidney dysfunction (eGFR: $<30 \mathrm{ml} \mathrm{min}^{-1}$ ), maintain the effect of diuretics

Guidance in salt intake restriction and use of diuretics according to the above principles

Combinations of antihypertensive drugs that have different action mechanisms, including a diuretic, at sufficient doses

Use of antihypertensive drugs at night or in the evening in patients with morning/nighttime hypertension

If non-steroidal anti-inflammatory drugs, adrenocorticosteroids, Kampo formulas containing licorice, oral contraceptives, cyclosporine, erythropoietin, antidepressants or molecule-targeting drugs are used concomitantly, discontinue them or reduce the dose if possible. Select antihypertensive drugs considering the pressor mechanisms of the other drugs and drug interactions

Screening tests should be conducted while monitoring of characteristic symptoms/findings. The patient should be referred to a hypertension specialist

Abbreviation: eGFR, estimated glomerular filtration rate.

proportion of patients in whom control with three or more antihypertensive drugs was unfavorable may not have been high.

With respect to the state of blood pressure control reported by clinicians/practitioners in Japan, the J-HOME Study (mean number of antihypertensive drugs used: 1.7) indicated that the proportion of patients with poorly controlled hypertension based on clinic blood pressure was $58 \%$, whereas that based on home blood pressure $\left(\geqslant 135 / 85 \mathrm{~mm} \mathrm{Hg}\right.$ ) was $66 \% .{ }^{513}$ In this study, both clinic and home blood pressures were controlled adequately in only 19\% of treated patients. The target control level of clinic blood pressure is $<130 /$ $80 \mathrm{~mm} \mathrm{Hg}$ in patients with diabetes mellitus, but it was achieved in only $11 \%$ according to another survey. ${ }^{514}$ Therefore, blood pressure is not controlled despite antihypertensive treatment in many patients, and the number of patients with resistant hypertension may not be small.

2) Factors for resistance to treatment and approaches to them In patients with resistant or poorly controlled hypertension, it is important to consider etiological factors and establish an appropriate approach. There are many factors for resistance to treatment, including failure to measure correct blood pressure (the use of a small cuff on a large arm, pseudohypertension (see Section 1 of Chapter 2, DIAGNOSIS OF HYPERTENSION)), blood pressure measurementassociated problems, such as white coat hypertension/white coat phenomenon, unfavorable adherence, lifestyle-related problems, such as excessive salt consumption, obesity and excessive alcohol consumption, patient conditions, such as sleep apnea syndrome and an excessive body fluid volume, combinations and doses of antihypertensive drugs, problems regarding the duration of drug efficacy, the consumption of drugs/foods that increase blood pressure or attenuate the actions of antihypertensive drugs and secondary hypertension (Table 5-3). Of these, white coat hypertension/white coat phenomenon, ${ }^{515}$ unfavorable adherence ${ }^{516}$ and sleep apnea syndrome ${ }^{517}$ are frequently observed, and the incidence of secondary hypertension is also high. Furthermore, physicians' problems including the inappropriate use of antihypertensive drugs are also involved in treatment resistance in many cases. ${ }^{518}$

In patients with apparent, white coat phenomenon-related resistant or poorly controlled hypertension, it may not be necessary to intensify antihypertensive treatment if home or 24-h blood pressure is controlled. In patients with white coat hypertension-type resistant hypertension, organ damage is less marked than in those with true resistant hypertension, and the prognosis is more favorable. ${ }^{515,519}$

Patient adherence and concordance are also important. If the patient does not accept antihypertensive treatment because of insufficient explanations, or if the physician is not aware of the adverse effects of antihypertensive drugs, adherence tends to be unsatisfactory. According to a survey of patients who were treated at outpatient hypertension clinics, patients in whom blood pressure control was favorable had a better understanding of antihypertensive treatment. ${ }^{520}$ Another survey regarding blood pressure control and its factors indicated that the attitude of physicians to treatment was the most important factor. ${ }^{518}$ To improve blood pressure control, a positive attitude of the physician to treatment, efforts to improve the patient's understanding of hypertension treatment, encouragement to modify lifestyle and the selection of appropriate antihypertensive drugs are important. The patient's economic and psychological problems must also be considered.

The resistance of hypertension is often ascribed to the volume overload that results from excessive salt intake, no use or inadequate use of diuretics and the presence of renal insufficiency. In this case, the appropriate use of diuretics is effective. Even in resistant hypertension patients taking antihypertensive drugs including diuretics, strict salt restriction is useful for decreasing blood pressure. ${ }^{521}$

If sufficient blood pressure control cannot be achieved, the presence or absence of the factors mentioned in Table 5-3 must be evaluated. If there is no sign of secondary hypertension or no problem with blood pressure measurement or drug compliance, but the control of blood pressure is insufficient even on treatment using three or more drugs, lifestyle guidance including salt restriction should be performed again.

For antihypertensive drug therapy, if no diuretic has been used, its use should be started, and, if a diuretic is used, its dose and type should be optimized. ${ }^{113}$ With respect to the adequate doses of 
diuretics, see the section regarding diuretics and a list of major drugs for hypertension. While monitoring the influence on electrolytes and metabolism, the use of a diuretic at a dose higher than the recommended dose can be considered. In patients with an eGFR of $30 \mathrm{ml} \mathrm{min}^{-1}$ or above, thiazide-type diuretics should be selected. When selecting trichlormethiazide, administration should be started at $1 \mathrm{mg}$ per day. If blood pressure control is insufficient, the dose can be increased to $2 \mathrm{mg}$ per day. In patients with an eGFR of $<30 \mathrm{ml} \mathrm{min}^{-1}$, loop diuretics should be selected. Among loop diuretics, furosemide has a short duration of action, and thus it must be administered several times a day. The use of a diuretic with a longer duration of action (such as torasemide) may be recommended.

Concerning antihypertensive drugs other than diuretics, several antihypertensive drugs differing in the action mechanism should be combined. When selecting these drugs, drugs to be positively indicated should be predominantly adopted, but a combination of a Ca channel blocker and an ARB or ACE inhibitor may be primarily used. Furthermore, it is important to use a sufficient dose for blood pressure control. It has been shown that combination therapy with a high-dose Ca channel blocker, a high-dose ARB and a standard-dose diuretic effectively decreases blood pressure in patients with severe or poorly controlled hypertension..$^{522}$

If a sufficient decrease in blood pressure cannot be achieved by combination therapy with three drugs, a Ca channel blocker, an ARB or ACE inhibitor and a diuretic, the pharmacological therapy should be intensified or modified. Increasing their doses, switching the frequency of dosing from once in the morning to twice in the morning and evening or once in the evening, and addition of another antihypertensive drug may be effective for blood pressure control (Table 5-4). In patients with resistant hypertension, if the duration of action of an antihypertensive drug is insufficient, a period of unfavorable blood pressure control is likely to occur. To control blood pressure at the target level over $24 \mathrm{~h}$, diurnal changes in blood pressure should be evaluated by morning and evening measurement of home blood pressure or 24-h ambulatory blood pressure monitoring, and not only the type of antihypertensive drugs but also the time of administration should be adjusted. A study showed that the administration of antihypertensive drugs before sleep decreased 24-h and nighttime blood pressures in patients with resistant hypertension, improving the proportion of patients in whom the target of blood pressure control is achieved. ${ }^{523}$

Concerning combination therapy, the efficacy of the additional administration of aldosterone antagonists has been reported. ${ }^{524,525}$ Sympatholytic drugs such as $\alpha \beta$-, $\alpha$ - and $\beta$-blockers may also be effective. If blood pressure control is unfavorable, additional combination therapy must be considered. Central sympatholytic drugs such as $\alpha$-methyldopa and vasodilators such as hydralazine become candidates. As a rule, the concomitant use of drugs of the same class should be avoided, but combination therapy with DHP and non-DHP Ca channel blockers, that with an ARB and an ACE or renin inhibitor and that with thiazide-type and loop diuretics may be selected. For such combination therapies or high-dose administration, adverse effects and an excessive decrease in blood pressure may occur, and caution is needed. Consultation with a hypertension specialist at an appropriate time is recommended for patients who require multiple antihypertensive drugs.

Recently, antihypertensive therapies by radiofrequency ablation of the renal nerves through a renal artery catheter or electric stimulation of baroreceptors through an electrode implanted into an area adjacent to the carotid sinus were developed. They have been shown to be effective for resistant hypertension. ${ }^{526}$ If these procedures are covered

\section{Table 5-4 Drug therapy for resistant or poorly controlled hypertension}

When the target level of blood pressure control cannot be achieved using three drugs, a Ca channel blocker, an ARB/ACE inhibitor and a diuretic,

1. Increasing the dose or changing the frequency of administration (to twice a day or once at night)

2. Additional administration of an aldosterone antagonist (the serum potassium level should be monitored)

3. Additional administration of a sympatholytic drug ( $\alpha \beta-, \beta$ - or $\alpha$-blockers)

4. Additional combination therapies

a. Addition of a centrally acting sympatholytic drug

b. Addition of a vasodilator (hydralazine, others)

c. Concomitant use of dihydropyridine and non-dihydropyridine Ca channel blockers

d. Concomitant use of two drugs from ARBs, ACE inhibitors and renin inhibitors (the serum potassium level and kidney function should be monitored)

e. Concomitant use of thiazide and loop diuretics

5. Consultation with a hypertension specialist at an appropriate time

Abbreviations: ACE, angiotensin-converting enzyme; ARB, angiotensin-receptor blocker

by health insurance, they may be applied as new treatment methods for resistant hypertension.

\section{3) Renal sympathetic denervation}

Renal sympathetic denervation (RDN) is the recently developed endovascular catheter technology to lower blood pressure. Via femoral artery access, both renal arteries are cannulated in sequence to generate high-frequency energy on the vascular wall and to ablate the renal nerves localized in the adventita of renal arteries. ${ }^{527-529}$ In 2009, RDN was first applied in humans for the treatment of resistant hypertension. ${ }^{527}$ To date, it is reported that the hypotensive effects of RDN had persisted for 2 years. ${ }^{528}$

As this minimally invasive procedure, $\mathrm{RDN}$, has a merit to selectively ablate the renal nerves alone, it is characterized by little adverse effects, ${ }^{527-529}$ differing from splanchnicectomy, which had been performed previously. ${ }^{530,531}$ The following limitations have been indicated in RDN: its effects on home and 24-h blood pressures are less marked than those on clinic blood pressure, and it is not always possible to reduce the number and doses of antihypertensive drugs. ${ }^{527-529,532,533}$ In current trials, subjects are limited to resistant hypertension with a systolic blood pressure exceeding $160 \mathrm{~mm} \mathrm{Hg}$ despite therapy using three or more antihypertensive drugs including a diuretic. RDN is contraindicated for patients with kidney dysfunction or anatomical abnormalities such as renal artery stenosis. ${ }^{533,534}$ Although a clinical trial also started in Japan, currently RDN is not covered by health insurance.

Once the long-term safety and good cardiovascular prognosis after RDN have been established, in addition to definite hypotensive effects from comparative studies with blind or drug therapy control groups, RDN may be a new confident treatment measure. Organ protection beyond blood pressure lowering is also expected with RDN. ${ }^{535}$

\section{Citation Information}

We recommend that any citations to information in the Guidelines are presented in the following format:

The Japanese Society of Hypertension Guidelines for the Management of Hypertension (JSH2014). Hypertens Res 2014; 37: 253-392.

Please refer to the title page for the full list of authors. 